\title{
Éclosion d'une forme invasive de Streptococcus pneumoniae dans les quartiers défavorisés de Victoria, en Colombie-Britannique, de 2016 à 2017
}

\author{
G McKee ${ }^{1 *}$, A Choi ${ }^{1}$, C Madill², J Marriott² ${ }^{2}$ P Kibsey², D Hoyano²
}

\section{Résumé}

Contexte : Bien que les pneumococcies invasives (PI) constituent une cause importante de morbidité et de mortalité, les éclosions sont relativement rares. Facteurs de risque connus, l'itinérance et la toxicomanie ont été associées à plusieurs éclosions au Canada, et ce, malgré les recommandations nationales qui ont été émises (vaccination régulière des enfants et vaccination antipneumococcique ciblée à l'âge adulte).

Objectif : Décrire l'épidémiologie et les problèmes de santé publique associés à l'éclosion d'une nouvelle forme de PI de sérotype 4 au sein d'une population de sans-abris et de personnes en situation de logement instable à Victoria, en Colombie-Britannique, au cours de l'automne et de l'hiver 2016-2017.

Résultats : On a entrepris une surveillance prospective améliorée pour les cas confirmés en laboratoire signalés à la santé publique, y compris les variables enregistrant la situation de logement et la toxicomanie. Entre le $1^{\text {er }}$ août 2016 et le $1^{\text {er }}$ septembre 2017, 33 cas de PI de sérotype 4 ont été déclarés à la santé publique, dans la région de Victoria. Par rapport aux autres sérotypes, ces cas étaient souvent des personnes d'âge moyen, sans-abXri ou en situation de logement instable, et qui avaient abusé récemment de la drogue ou de l'alcool. Bien qu'on ait lancé une campagne ciblée de vaccination antipneumococcique en collaboration avec des organismes communautaires externes, ces initiatives ont souffert de l'incomplétude des données et de problèmes de dotation en personnel.

Conclusion : Le présent article illustre une éclosion de PI de sérotype 4 au sein d'une population habitant les quartiers défavorisés et comportant de nombreux facteurs de risque, dont l'itinérance, une situation de logement instable et la toxicomanie. En raison des défis associés au contrôle de cette éclosion, il est essentiel d'accroître la capacité de dépistage et la couverture vaccinale parmi cette population marginalisée.

\section{Affiliations}

${ }^{1}$ School of Population and Public Health, Université de la Colombie-Britannique, Vancouver (Colombie-Britannique)

2 Island Health, Victoria (Colombie-Britannique)

*Correspondance : gwmckee@alumni.ubc.ca

Citation proposée : McKee G, Choi A, Madill C, Marriott J, Kibsey P, Hoyano D. Éclosion d'une forme invasive de Streptococcus pneumoniae dans les quartiers défavorisés de Victoria, en Colombie-Britannique, de 2016 à 2017. Relevé des maladies transmissibles au Canada 2018;44(12):360-6. https://doi.org/10.14745/ccdr. v44i12a02f

Mots-clés : Pneumococcies, éclosions, sans-abris, toxicomanes, Colombie-Britannique, Canada

\section{Introduction}

La pneumococcie invasive $(\mathrm{PI})$ résulte de l'infection d'un site normalement stérile par la bactérie à Gram positif Streptococcus pneumoniae (1). Se manifestant d'habitude sous la forme d'une pneumonie, d'une méningite ou d'une bactériémie, elle présente, au Canada, une incidence globale variant entre 8,9 et
9,9 cas pour 100000 personnes (2). Quoique les taux chez les nourrissons aient décliné sensiblement après la mise en œuvre de la vaccination régulière des enfants, les taux sont demeurés essentiellement les mêmes chez les adultes. La PI continue de représenter une source importante de morbidité et de mortalité, 
surtout chez les personnes à risque dont la couverture vaccinale n'est pas complète (2).

Les sans-abris et les personnes à faible revenu dans les quartiers défavorisés constituent des exemples de populations très vulnérables. Bien que la plupart des cas de PI soient sporadiques et que les (rares) éclosions surviennent surtout dans des établissements "fermés ", on a signalé un certain nombre d'éclosions communautaires dans les quartiers défavorisés de I'Ouest canadien (3-5).

En 2008, le Comité consultatif national de l'immunisation (CCNI) a déterminé qu'il existait un nombre suffisamment de données probantes pour conclure que les sans-abris étaient plus vulnérables aux PI. Cependant, on ne sait pas si cette association constituait un lien causal ou si elle reflétait plutôt les facteurs de risque qui se présentent de manière disproportionnée chez les sans-abris $(3,5-7)$.

Étant donné que les sérotypes associés à ces éclosions comptent parmi les sérotypes que combattent des vaccins antipneumococciques largement disponibles, il existe de nombreuses possibilités d'intervention (8). Le CCNI recommande $d$ 'administrer le vaccin antipneumococcique polysaccharidique 23-valent aux sans-abris et aux toxicomanes. II est cependant difficile de joindre cette population, notamment parce qu'elle est souvent transitoire.

La population de Victoria s'élève à quelque 86000 personnes, dont 1700 sont sans abri ou mal logées $(9,10)$. Une grande partie des sans-abris de Victoria ne sont que de passage, $28 \%$ ayant déménagé dans cette ville il y a moins d'un an (9). Les services de proximité, d'infirmier(ère)s de rue et d'infirmier(ère) s en santé publique sont prodigués par Island Health, I'une des cinq régies régionales de la santé de la Colombie-Britannique. Cependant, la capacité de ces services de réduire les méfaits et de prévenir également les maladies transmissibles est devenue limitée. La hausse de l'itinérance, les problèmes de dotation et les conflits de priorités créés par l'épidémie de surdose d'opioïdes ont causé des problèmes supplémentaires, ce qui complique l'adoption de mesures devant l'éclosion de PI de sérotype 4 à Victoria, détectée à l'automne 2016.

Le présent article a pour objectif de décrire l'épidémiologie d'une éclosion de PI communautaire de sérotype 4 chez des sans-abris et des personnes en situation de logement instable à Victoria ainsi que les défis liés aux enquêtes de santé publique et aux interventions dans le même domaine.

\section{Méthodologie}

\section{Détection de l'éclosion}

En août 2016, les infirmier(ère)s spécialisé(e)s en maladies transmissibles qui reçoivent régulièrement des avis de maladies à déclaration obligatoire ont observé une augmentation des cas de PI de sérotype 4 (surtout des pneumonies) et les médecins microbiologistes de l'hôpital local ont déclaré un nombre anormalement élevé de sans abri provenant des quartiers défavorisés parmi les patients atteints d'une PI admis à l'unité des soins intensifs. On a constaté une hausse des cas de PI grâce aux alertes de surveillance de routine régionales, qui comparent le nombre de cas de maladies à déclaration obligatoire aux moyennes historiques sur cinq ans, et grâce au système provincial qui lance des alertes après avoir constaté des écarts statistiques entre les données observées et les données historiques. Selon la surveillance régionale, le nombre de cas de PI signalés en septembre 2016 était huit fois plus important que la moyenne mensuelle sur cinq ans, tandis que, en octobre 2016, on a enregistré une hausse de $300 \%$. À la lumière de tous ces indices, le médecin hygiéniste a déclaré en octobre 2016 qu'on se trouvait en présence d'une éclosion de PI de sérotype 4.

\section{Enquête}

La PI est une maladie à déclaration obligatoire en Colombie-Britannique depuis 1999 et au Canada depuis 2000 (1,11). En Colombie-Britannique, la PI est définie par la manifestation clinique d'une maladie invasive et l'obtention d'une confirmation en laboratoire de Streptococcus pneumoniae dans un site normalement stérile, comme le sang ou le liquide céphalorachidien, à l'exception de l'oreille moyenne (12).

Tous les échantillons ont été prélevés dans les locaux d'Island Health. C'est I'hôpital Royal Jubilee de Victoria qui a effectué la culture des isolats de $S$. pneumoniae et en a évalué la sensibilité aux médicaments et c'est le Laboratoire national de microbiologie qui a procédé au sérotypage plus spécifique. Les médecins biologistes ont signalé à la santé publique des cas détectés répondant à la définition d'une PI. Les données de tous les cas de PI dans la région (zone de prestation de services de santé du sud de l'île d'Island Health) ont été versées dans Microsoft Excel (Corporation Microsoft, Redmond, Washington, États-Unis), après avoir été recueillies sur des cahiers de recherche formalisés servant à la surveillance de routine et sur des dossiers médicaux électroniques.

On a ajouté à ces cahiers de nouvelles variables (des facteurs de risque), y compris la situation de logement et la toxicomanie. Même si l'on n'a pas communiqué directement avec les patients afin d'obtenir d'eux des renseignements additionnels, on a procédé à un examen rétrospectif de leur état de santé au moyen de leur dossier de santé électronique lorsqu'ils avaient été soignés par un établissement de santé publique, de leurs visites à la salle des urgences, de leurs admissions à I'hôpital, des enquêtes sur les malades externes et des profils cliniques sur la santé mentale et la toxicomanie. Les dossiers des cas ont été examinés par deux des auteurs (GM et $A C$ ) et les données ont été encodées à l'aide des définitions normalisées (annexe 1). On a stratifié les cas par sérotype (sérotype 4 ou un autre sérotype) afin de procéder à l'analyse descriptive en Excel. On a comparé les variables continues à l'aide du test de Student (échantillons indépendants) en prenant comme hypothèse que les variances 
étaient différentes. On a comparé les variables nominales en employant la méthode exacte de Fisher $\left(\mathrm{X}^{2}\right)$, selon la taille de la cellule. Enfin, on a calculé les rapports de cotes non ajustés à l'aide de la régression logistique du logiciel statistique $R(R$ Foundation, Vienne, Autriche).

\section{Résultats}

Entre le $1^{\text {er }}$ août 2016 et le 1er septembre 2017, on a signalé à la santé publique un total de 84 cas de PI dans la zone de prestation de services de santé du sud de l'île. Alors que seulement trois cas de $S$ pneumoniae de sérotype 4 avaient

Figure 1A : Distribution sérotypique des isolats de Streptococcus pneumoniae chez des patients atteints de pneumococcie invasive dans la zone de prestation de services de santé du sud de l'île (Colombie-Britannique, Canada), du $1^{\text {er }}$ janvier 2012 au 31 juillet 2016

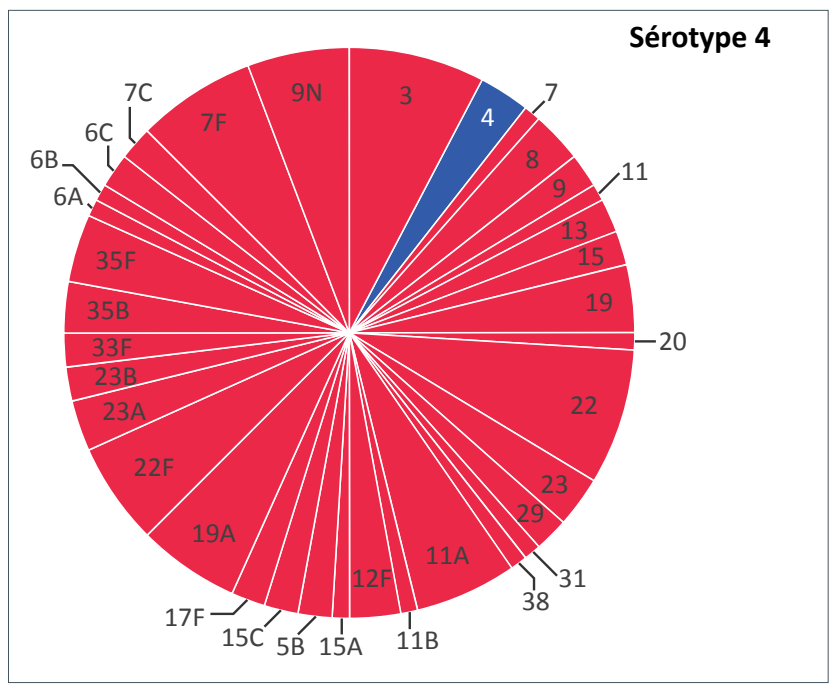

été déclarés au cours des 4,5 années précédentes, 33 ont été signalés au cours de l'étude, ce qui représente $39,3 \%$ de tous les cas signalés (figures $1 \mathrm{~A}$ et 1B).

Les déclarations de cas atteints du sérotype 4 ont atteint un sommet en septembre et en octobre 2016 et ont persisté tout au long de la période visée par l'étude avant de décliner en mars 2017 (figure 2).

On compare au tableau 1 les profils démographiques et les profils de risque des cas de PI de sérotype 4 et d'autres sérotypes signalés au cours de la période visée par l'étude.

Figure 1B : Distribution sérotypique des isolats de Streptococcus pneumoniae chez des patients atteints de pneumococcie invasive dans la zone de prestation de services de santé du sud de l'île (Colombie-Britannique, Canada), du 1 ${ }^{\text {er }}$ août 2016 au 1er septembre 2017

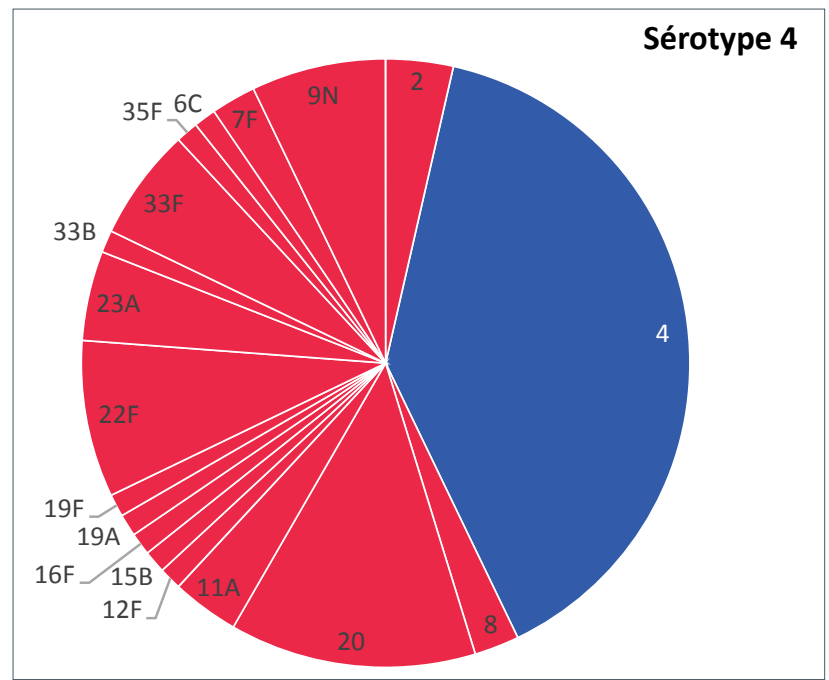

Figure 2 : Comparaison du nombre de cas signalés de pneumococcie invasive de sérotype 4 et d'un autre sérotype avec la moyenne mensuelle sur cinq ans de tous les sérotypes, zone de prestation de services de santé du sud de l'île, du 1 er janvier 2016 au 1 er septembre 2017

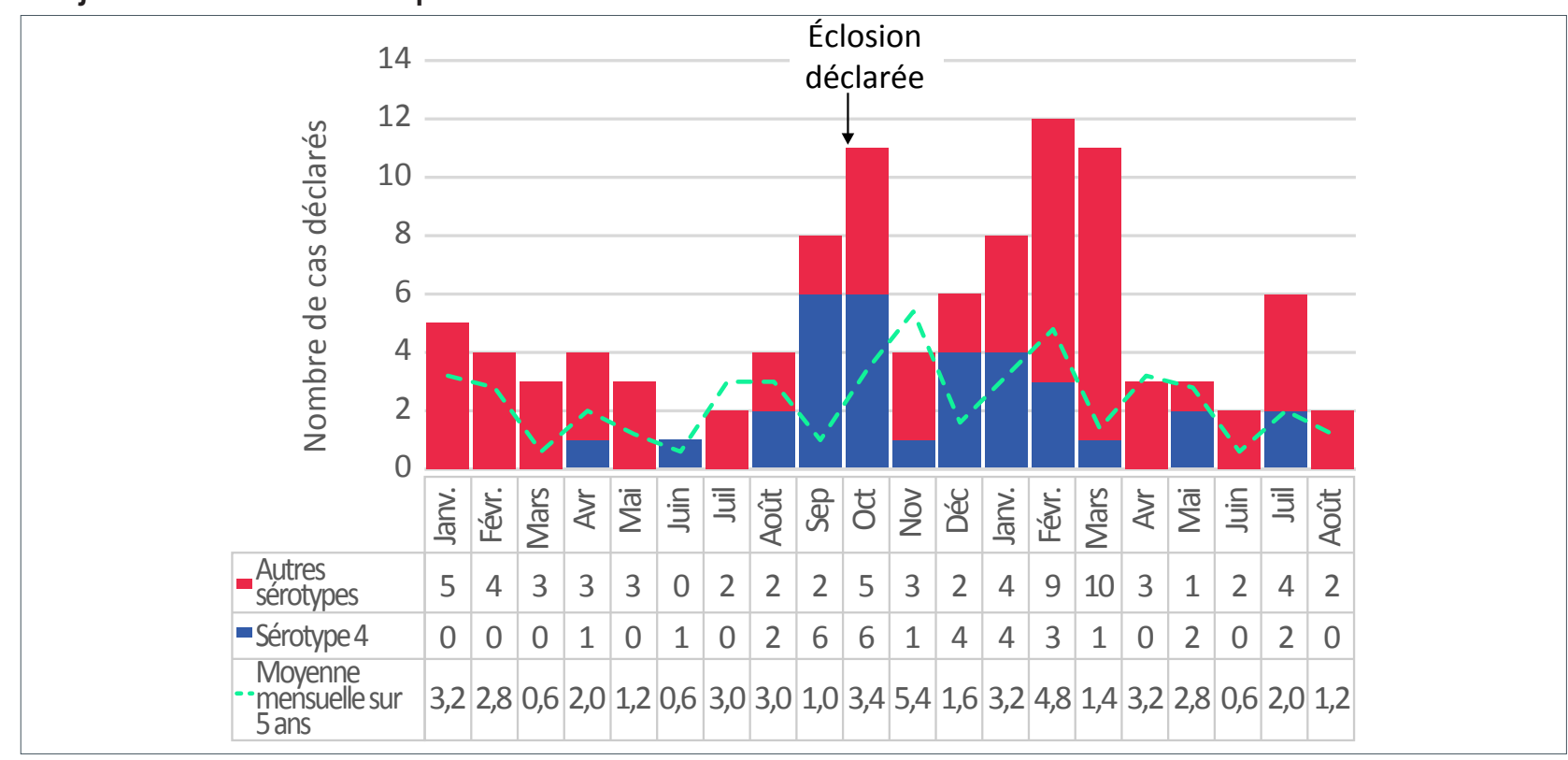


Tableau 1 : Données démographiques, caractéristiques et résultats chez les cas atteints de pneumococcie invasive de sérotype 4 et d'autres sérotypes dans la zone de prestation de services de santé du sud de l'île d'Island Health (Colombie-Britannique, Canada), du $1^{\text {er }}$ août 2016 au $1^{\text {er }}$ septembre 2017

\begin{tabular}{|c|c|c|c|c|c|}
\hline \multirow{2}{*}{ Variable } & \multicolumn{2}{|c|}{ Sérotype 4} & \multicolumn{2}{|c|}{ Autres } & \multirow{2}{*}{$\begin{array}{c}\text { valeur } \\
p\end{array}$} \\
\hline & $n$ & $\%$ & $\mathrm{n}$ & $\%$ & \\
\hline Nombre total de cas & 33 & 100 & 51 & 100 & N/A \\
\hline \multicolumn{6}{|l|}{ Sexe } \\
\hline Sexe masculin & 23 & 69,70 & 29 & 56,86 & 0,34 \\
\hline \multicolumn{6}{|l|}{ Situation de logement } \\
\hline $\begin{array}{l}\text { Sans abri ou en } \\
\text { situation de logement } \\
\text { instable }\end{array}$ & 16 & 48,48 & 8 & 15,69 & $<0,01$ \\
\hline \multicolumn{6}{|l|}{ Alcoolisme avancé } \\
\hline Alcoolisme avancé & 13 & 39,39 & 16 & 31,37 & 0,80 \\
\hline $\begin{array}{l}\text { Consommation de } \\
\text { drogues injectables }\end{array}$ & 10 & 30,30 & 5 & 9,80 & 0,054 \\
\hline Cocaïne & 8 & 24,24 & 5 & 9,80 & 0,20 \\
\hline Méthamphétamine & 10 & 30,30 & 3 & 5,88 & $<0,05$ \\
\hline Cannabis & 18 & 54,55 & 8 & 15,69 & $<0,01$ \\
\hline Opioïdes & 18 & 54,55 & 7 & 13,73 & $<0,001$ \\
\hline Tabagisme & 27 & 81,82 & 18 & 35,29 & $<0,001$ \\
\hline \multicolumn{6}{|l|}{ Comorbidités } \\
\hline Virus de I'hépatite C & 7 & 21,21 & 9 & 17,65 & 1,00 \\
\hline $\mathrm{VIH}$ & 1 & 3,03 & 3 & 5,88 & 0,64 \\
\hline Maladie pulmonaire & 9 & 27,27 & 23 & 45,10 & 0,086 \\
\hline $\begin{array}{l}\text { Maladie } \\
\text { cardiovasculaire }\end{array}$ & 5 & 15,15 & 21 & 41,18 & $<0,05$ \\
\hline Maladie rénale & 1 & 3,03 & 13 & 25,49 & $<0,01$ \\
\hline Diabète & 1 & 3,03 & 9 & 17,65 & $<0,05$ \\
\hline \multicolumn{6}{|c|}{ Présentation de la pneumococcie invasive } \\
\hline Pneumonie & 28 & 84,85 & 43 & 84,31 & 1,00 \\
\hline Méningite & 2 & 6,06 & 3 & 5,88 & 1,00 \\
\hline \multicolumn{6}{|l|}{ Niveau de soins } \\
\hline Hospitalisation & 31 & 93,94 & 46 & 90,20 & 1,00 \\
\hline $\begin{array}{l}\text { Admission à l'unité des } \\
\text { soins intensifs }\end{array}$ & 10 & 30,30 & 12 & 23,53 & 0,70 \\
\hline \multicolumn{6}{|l|}{ Résultats } \\
\hline Décès à l'hôpital & 0 & 0 & 10 & 19,61 & $<0,01$ \\
\hline \multicolumn{6}{|l|}{ Immunisation } \\
\hline $\begin{array}{l}\text { Vaccination } \\
\text { antipneumococcique } \\
\text { antérieure }^{\mathrm{a}}\end{array}$ & 3 & 9,09 & 8 & 15,69 & 0,52 \\
\hline
\end{tabular}

Abréviations : $n$, nombre; s. o., sans objet; $<$, inférieur à

a On a déterminé si le sujet avait été vacciné contre les pneumococcies en étudiant son dossier électronique. Comme ces dossiers sont incomplets, il faut faire preuve de prudence en interprétant ces chiffres

Note: Les chiffres sont arrondis à la décimale près
On n'a constaté aucune différence significative de la distribution selon le sexe entre les deux groupes, les deux types de sérotype infectant surtout les hommes. L'âge médian des cas atteints par le sérotype 4 (médiane $=46$ ans, écart-type $=15,22$ ans) était sensiblement plus bas $(p<0,001)$ que pour les cas atteints par les autres sérotypes (médiane $=63$ ans, écart-type $=18,21$ ans). Les personnes de plus de 75 ans ont été exclues. Les cas atteints par le sérotype 4 étaient beaucoup plus susceptibles d'être sans abri ou en situation de logement instable $(48,48 \%$ par rapport à $15,69 \%$ ). Si la toxicomanie était plus prévalente chez les cas atteints du sérotype 4 , on n'a constaté de différences significatives que pour la méthamphétamine, le cannabis, les opioïdes et le tabac.

Les cas atteints du sérotype 4 présentaient généralement moins de comorbidités que pour les autres sérotypes, à l'exception importante des maladies cardiovasculaires, des maladies rénales et du diabète. Bien qu'on n'ait observé aucune différence sensible dans la présentation clinique, l'hospitalisation ou l'admission aux soins intensifs, on a constaté 10 décès à l'hôpital chez les patients atteints des autres sérotypes et aucun décès à I'hôpital chez ceux atteints du sérotype 4 .

Comme le montre le tableau 2, I'analyse bivariée des facteurs de risque a illustré encore plus clairement les différences entre le sérotype 4 et les autres sérotypes. Comme on a pu le voir dans l'analyse descriptive, les probabilités que les cas atteints par le sérotype 4 soient sans abri ou en situation de logement instable étaient 4,82 (intervalle de confiance [IC] de $95 \%: 1,79$ à 13,97) fois plus élevées que pour les autres sérotypes. Ces cas étaient également plus susceptibles d'être atteints de toxicomanies : utilisation de drogues par injection (rapport de cotes $[R C]: 3,65$; IC de $95 \%: 1,15$ à 12,95), utilisation de méthamphétamine (RC: 6,23; IC de $95 \%: 1,71$ à 29,89), utilisation de cannabis (RC: 5,85 ; IC de $95 \%: 2,17$ à 17,07), utilisation d'opioïdes (RC : 6,69; IC de $95 \%: 2,41$ à 20,36) et tabagisme (RC: 7,25 ; IC de $95 \%$ : 2,64 à 22,62 ). On a observé des associations négatives pour les maladies cardiovasculaires (RC: 0,$22 ; \mathrm{IC}$ de $95 \%: 0,07$ à 0,63 ) et rénales ( $R C: 0,08$; IC de $95 \%: 0$ à 0,45 ).

\section{Intervention des services de santé publique}

En réponse à l'éclosion, le programme de infirmier(ère)s de rue d'Island Health a lancé une campagne visant à accroître l'administration du vaccin polysaccharidique 23 -valent contenant le sérotype 4, qui a été fortement encouragée par la collaboration avec plusieurs fournisseurs de services dans les quartiers défavorisés qui avaient déjà noué des relations avec les personnes les plus vulnérables à l'infection. D'août 2016 à septembre 2017, des infirmier(ère)s de rue ont administré une centaine de doses, tandis que plus de 80 doses ont été administrées par d'autres prestataires de services.

En avril 2017, le nombre total de cas de PI déclarés avait décliné à des niveaux comparables à la moyenne. Après plusieurs mois où le nombre de cas signalés est demeuré systématiquement peu élevé, on a conclu qu'il n'était plus nécessaire de procéder 
à une surveillance accrue. Précisons toutefois que la proportion de cas de PI de sérotype 4 est restée supérieure aux niveaux constatés avant l'éclosion, ce qui laisse croire à une circulation faible mais persistante.

Tableau 2 : Analyse bivariée des facteurs de risque chez les cas atteints de pneumococcie invasive de sérotype 4 et d'autres sérotypes dans la zone de prestation de services de santé du sud de l'île (Colombie-Britannique, Canada), du 1er août 2016 au 1er septembre 2017

\begin{tabular}{|l|r|r|r|}
\hline Facteurs de risque & RC & IC de 95 \% & Valeur $p$ \\
\hline $\begin{array}{l}\text { Sans abri ou en } \\
\text { situation de logement } \\
\text { instable }\end{array}$ & 4,82 & {$[1,79$ à 13,97$]$} & $<0,01$ \\
\hline Alcoolisme avancé & 1,26 & {$[0,5$ à 3,18$]$} & 0,62 \\
\hline $\begin{array}{l}\text { Consommation de } \\
\text { drogues injectables }\end{array}$ & 3,65 & {$[1,15$ à 12,95$]$} & $<0,05$ \\
\hline Cocaïne & 2,62 & {$[0,79$ à 9,53$]$} & 0,12 \\
\hline Méthamphétamine & 6,23 & {$[1,71$ à 29,89$]$} & $<0,01$ \\
\hline Cannabis & 5,85 & {$[2,17$ à 17,07$]$} & $<0,001$ \\
\hline Opioïdes & 6,69 & {$[2,41$ à 20,36$]$} & $<0,001$ \\
\hline Tabagisme & 7,25 & {$[2,64$ à 22,62$]$} & $<0,001$ \\
\hline Virus de l'hépatite C & 1,17 & {$[0,37$ à 3,52$]$} & 0,78 \\
\hline VIH & 0,47 & {$[0,02$ à 3,85$]$} & 0,52 \\
\hline Maladie pulmonaire & 0,39 & {$[0,15$ à 1,00$]$} & 0,054 \\
\hline $\begin{array}{l}\text { Maladie } \\
\text { cardiovasculaire }\end{array}$ & 0,22 & {$[0,07$ à 0,63$]$} & $<0,01$ \\
\hline Maladie rénale & 0,08 & {$[0$ à 0,45$]$} & $<0,05$ \\
\hline Diabète & 0,13 & {$[0,01$ à 0,76$]$} & 0,06 \\
\hline
\end{tabular}

Abréviations : IC, intervalle de confiance; $\mathrm{RC}$, rapport de cotes; $<$, inférieur à

\section{Discussion}

Malgré l'endémicité chronique des pneumococcies au Canada, les éclosions de PI sont relativement rares. Bien que le sérotype 4 ait la réputation d'être très invasif, certaines études laissent croire que son invasivité aurait beaucoup plus à voir avec la composition de son enveloppe que son sérotype (13). N'empêche que des sérotypes invasifs sont souvent responsables des éclosions de $\mathrm{Pl}$, comme les éclosions de sérotype 5 au Canada $(3,5)$. Le présent article enrichit la documentation limitée sur les éclosions de PI de sérotype 4. Compte tenu des caractéristiques des sans-abris touchés par cette éclosion et de l'état des connaissances sur les risques associés aux $\mathrm{Pl}$, il peut être indiqué de renforcer les programmes de prévention ciblés.

Le fait que le sérotype responsable de l'éclosion soit évitable par la vaccination (4) remet en question l'approche actuellement adoptée pour l'administration des vaccins recommandés à la population à risque. Malgré la recommandation du CCNI, soit $d^{\prime}$ offrir des vaccins antipneumococciques aux sans-abris et aux personnes atteintes de toxicomanie, ces populations sont souvent difficiles à joindre. La capacité limitée des infirmier(ère)s de rue à servir la population des quartiers défavorisés constituait un problème avant et pendant l'éclosion. En plus de ses difficultés de dotation, ce service souffrait du conflit de priorités associé aux mesures à prendre devant la crise des opioïdes. II s'ensuit que la transmission du pneumocoque peut avoir été exacerbée par la réduction, l'année précédente, du nombre de vaccinations antipneumococciques ciblées, causée par cette capacité limitée dans la région de Victoria. Ayant reconnu ces lacunes pendant l'éclosion, on a ajouté un poste afin d'améliorer le service.

La campagne de vaccination antipneumococcique ciblée constituait un volet essentiel de la réponse des services de santé publique à l'éclosion de PI. Il est difficile de déterminer le degré auquel les efforts déployés pour élargir la vaccination ont contribué à la réduction observée des nouveaux cas. Des campagnes de vaccination semblables, visant à contrôler les éclosions de cette infection, ont obtenu des résultats mitigés $(3,5)$.

Au cours de l'éclosion, on a relevé l'existence de plusieurs obstacles à la vaccination ciblée, dont l'incomplétude des dossiers de vaccination, ce qui a rendu difficile la tâche d'identifier les personnes à vacciner. Bien que les employés de la santé publique et les fournisseurs de services communautaires de la régie régionale de la santé administrent tous deux des vaccins, ils ont recours à des systèmes d'information différents. L'intégration des dossiers médicaux et des dossiers de vaccination aurait pu améliorer l'évaluation des statuts de vaccination individuels et l'évaluation de l'efficacité du vaccin au sein de la population.

En plus des difficultés de dotation et de l'incomplétude des dossiers de vaccination, d'autres problèmes ont limité la portée de l'enquête. Étant donné que l'analyse des facteurs de risque des cas reposait sur l'examen rétrospectif des dossiers, il se peut que la sous-déclaration de ces facteurs ait introduit un biais statistique, en l'occurrence une erreur de classement. Même si l'absence de suivi direct des cas de PI constitue la pratique courante pour les infirmier(ère)s de santé publique de la régie régionale de la santé, l'interrogation des patients aurait fourni des détails supplémentaires, ce qui aurait mieux fait comprendre le mode de transmission de la maladie. À l'avenir, une analyse plus poussée des réseaux de contacts pourrait aider à reconnaître les éventuels sites de transmission, un certain refuge ou lieu de rencontre, ce qui pourrait éclairer la prise de mesures ciblées en santé publique.

\section{Conclusion}

Le présent article illustre une éclosion de PI de sérotype 4 au sein d'une population habitant les quartiers défavorisés et comportant de nombreux facteurs de risque de transmission, dont l'itinérance, une situation de logement instable et la toxicomanie. En outre, il illustre le besoin chronique d'améliorer l'approche communautaire et l'administration de vaccins antipneumococciques à cette population marginalisée. 


\section{Déclaration des auteurs}

Tous les auteurs ont participé à la conceptualisation, à l'analyse et à l'interprétation des données, ainsi qu'à la rédaction de l'ébauche du présent article. GM, AC, CM et JM ont aussi participé à la collecte des données.

\section{Conflit d'intérêt}

Aucun.

\section{Remerciements}

Nous tenons à remercier Island Health et les partenaires communautaires, qui ont joué un rôle majeur dans le contrôle de cette éclosion en prodiguant des soins et un soutien social tout en contribuant à la campagne de vaccination, ainsi que le Laboratoire national de microbiologie pour le rôle qu'il a joué dans le sérotypage des échantillons. Nous voulons aussi remercier Angela Reid, analyste de la surveillance à Island Health, pour le soutien qu'elle a accordé à la collecte de données, et Dre Monika Naus, directrice médicale du Communicable Diseases \& Immunization Service du BC Centre for Disease Control, qui a révisé le présent article.

\section{Références}

1. Gouvernement du Canada. Pneumococcies invasives. Ottawa (ON): Agence de la santé publique du Canada, Santé Canada; 2016. https://www.canada.ca/fr/sante-publique/ services/immunisation/maladies-pouvant-etre-prevenuesvaccination/pneumococcies-invasives/professionels.html

2. Gouvernement du Canada. Maladies évitables par la vaccination : Rapport de surveillance en date du 31 décembre 2015. Ottawa (ON): Agence de la santé publique du Canada; 2017. https://www.canada.ca/fr/sante-publique/ services/publications/vie-saine/maladies-evitable s-vaccination-rapport-surveillance-31-decembre-2015.html

3. Romney MG, Hull MW, Gustafson R, Sandhu J, Champagne S, Wong T, Nematallah A, Forsting S, Daly P. Large community outbreak of Streptococcus pneumoniae serotype 5 invasive infection in an impoverished, urban population. Clin Infect Dis 2008 Sep;47(6):768-74. DOI PubMed

4. Schillberg E, Isaac M, Deng X, Peirano G, Wylie JL, Van Caeseele P, Pillai DR, Sinnock H, Mahmud SM. Outbreak of invasive Streptococcus pneumoniae serotype 12F among a marginalized inner-city population in Winnipeg, Canada, 2009-2011. Clin Infect Dis 2014 Sep;59(5):651-7. DOI PubMed

5. Vanderkooi OG, Church DL, MacDonald J, Zucol F, Kellner JD. Community-based outbreaks in vulnerable populations of invasive infections caused by Streptococcus pneumoniae serotypes 5 and 8 in Calgary, Canada. PLoS One 2011;6(12):e28547. DOI PubMed

6. Comité consultatif national de l'immunisation (CCNI). Déclaration sur les usages recommandés du vaccin 23-valent contre le pneumocoque chez les sans-abri et les utilisateurs de drogues par injection. Une déclaration d'un comité consultatif (DCC). Relevé des maladies transmissibles au Canada 2008 Sep;34 DCC-5:1-12.

7. Cruickshank HC, Jefferies JM, Clarke SC. Lifestyle risk factors for invasive pneumococcal disease: a systematic review. BMJ Open 2014 Jun;4(6):e005224. DOl PubMed

8. Merck Sharpe \& Dohme Corp. Pneumococcal Serotypes. 2017. www.merckvaccines.com/Products/Pneumovax/2 3-pneumococcal-serotypes

9. Albert M, Penna T, Pagan F, Pauly B. More Than a Number: 2016 Greater Victoria Point in Time Count Summary Report. Victoria (BC); Employment and Social Development Canada; 2016. https://www.crd.bc.ca/docs/default-source/ housing-pdf/pitcount-report26apr2016.pdf

10. Government of British Columbia. Population Estimates. Victoria (BC); BC Stats; 2018. www2.gov.bc.ca/gov/content/ data/statistics/people-population-community/population/ population-estimates

11. BC Centre for Disease Control. List of Reportable Communicable Diseases in BC, July 2009. Vancouver (BC); BC CDC: 2009. http://www.bccdc.ca/resource-gallery/ Documents/Guidelines\%20and\%20Forms/Guidelines\%20 and\%20Manuals/Epid/Other/Epid_Guidelines_reportable_ diseases_British_Columbia_July2009.pdf

12. BC Centre for Disease Control. Streptococcus pneumonia, invasive - Case Definition. Vancouver (BC); BC CDC: 2017. http://www.bccdc.ca/health-professionals/clinical-resources/ case-definitions/pneumococcal-disease-invasive

13. Hausdorff WP, Feikin DR, Klugman KP. Epidemiological differences among pneumococcal serotypes. Lancet Infect Dis 2005 Feb;5(2):83-93. DOI PubMed 


\section{Annexe}

\section{Tableau 1 : Définition des variables originales servant à l'analyse}

\begin{tabular}{|c|c|}
\hline Variable & Définition \\
\hline Âge & $\begin{array}{l}\text { Âge du patient lorsqu'il a été hospitalisé pour une pneumococcie invasive, figurant dans son dossier médical } \\
\text { électronique }\end{array}$ \\
\hline Sexe & Sexe figurant dans le dossier médical électronique du patient \\
\hline $\begin{array}{l}\text { Sans abri ou en situation de } \\
\text { logement instable }\end{array}$ & $\begin{array}{l}\text { Sans adresse fixe OU identifié comme un sans-abri, mal logé OU faisant appel au service loge-trotteurs OU } \\
\text { habitant une tente ou un refuge, selon son dossier médical électronique }\end{array}$ \\
\hline \multicolumn{2}{|l|}{ Toxicomanie } \\
\hline Alcoolisme avancé & $\begin{array}{l}\text { Abus d'alcool OU consommation dépassant la quantité recommandée dans les lignes directrices pour la } \\
\text { consommation d'alcool à faible risque, selon le dossier médical électronique du patient }\end{array}$ \\
\hline $\begin{array}{l}\text { Consommation de drogues } \\
\text { injectables }\end{array}$ & Consommation de drogues injectables figurant dans le dossier médical électronique du patient \\
\hline Cocaïne & $\begin{array}{l}\text { Consommation de cocaïne décrite dans les commentaires du dossier OU détectée au moyen d'un test de } \\
\text { dépistage toxicologique au cours de l'année précédente, selon le dossier médical électronique du patient }\end{array}$ \\
\hline Méthamphétamine & $\begin{array}{l}\text { Consommation de méthamphétamine décrite dans les commentaires du dossier OU détectée au moyen d'un } \\
\text { test de dépistage toxicologique au cours de l'année précédente, selon le dossier médical électronique du } \\
\text { patient }\end{array}$ \\
\hline Cannabis & $\begin{array}{l}\text { Consommation de cannabis décrite dans les commentaires du dossier OU détectée au moyen d'un test de } \\
\text { dépistage toxicologique au cours de l'année précédente, selon le dossier médical électronique du patient }\end{array}$ \\
\hline Opioïdes & $\begin{array}{l}\text { Consommation d'opiö̈des illicites décrite dans les commentaires du dossier OU détectée au moyen d'un test } \\
\text { de dépistage toxicologique au cours de l'année précédente, selon le dossier médical électronique du patient }\end{array}$ \\
\hline Tabagisme & $\begin{array}{l}\text { Action d'avoir fumé du tabac au cours de l'année précédente, décrite dans les commentaires du dossier, selon } \\
\text { le dossier médical électronique du patient }\end{array}$ \\
\hline Alcoolisme avancé & $\begin{array}{l}\text { Abus d'alcool OU consommation dépassant la quantité recommandée dans les lignes directrices pour la } \\
\text { consommation d'alcool à faible risque, selon le dossier médical électronique du patient }\end{array}$ \\
\hline $\begin{array}{l}\text { Consommation de drogues } \\
\text { injectables }\end{array}$ & Consommation de drogues injectables figurant dans le dossier médical électronique du patient \\
\hline Cocaïne & $\begin{array}{l}\text { Consommation de cocaïne décrite dans les commentaires du dossier OU détectée au moyen d'un test de } \\
\text { dépistage toxicologique au cours de l'année précédente, selon le dossier médical électronique du patient }\end{array}$ \\
\hline Méthamphétamine & $\begin{array}{l}\text { Consommation de méthamphétamine décrite dans les commentaires du dossier OU détectée au moyen d'un } \\
\text { test de dépistage toxicologique au cours de l'année prédécente, selon le dossier médical électronique du } \\
\text { patient }\end{array}$ \\
\hline Cannabis & $\begin{array}{l}\text { Consommation de cannabis décrite dans les commentaires du dossier OU détectée au moyen d'un test de } \\
\text { dépistage toxicologique au cours de l'année précédente, selon le dossier médical électronique du patient }\end{array}$ \\
\hline Opioïdes & $\begin{array}{l}\text { Consommation d'opiö̈des illicites décrite dans les commentaires du dossier OU détectée au moyen d'un test } \\
\text { de dépistage toxicologique au cours de l'année précédente, selon le dossier médical électronique du patient }\end{array}$ \\
\hline Tabagisme & $\begin{array}{l}\text { Action d'avoir fumé du tabac au cours de l'année précédente, décrite dans les commentaires du dossier, selon } \\
\text { le dossier médical électronique du patient }\end{array}$ \\
\hline \multicolumn{2}{|c|}{ Présentation de la pneumococcie invasive } \\
\hline Pneumonie & Pneumonie pneumococcique mentionnée dans le dossier médical électronique du patient \\
\hline Méningite & Méningite pneumococcique mentionnée dans le dossier médical électronique du patient \\
\hline \multicolumn{2}{|l|}{ Niveau de soins } \\
\hline Hospitalisation & $\begin{array}{l}\text { Admission à l'hôpital pour une pneumococcie invasive, mentionnée dans le dossier médical électronique du } \\
\text { patient }\end{array}$ \\
\hline Durée de l'hospitalisation & $\begin{array}{l}\text { Nombre de jours entre la date de l'admission pour une pneumococcie invasive (dans la zone desservie par } \\
\text { Island Health) et la date du décès du patient ou de son congé de l'hôpital }\end{array}$ \\
\hline $\begin{array}{l}\text { Admission à l'unité des soins } \\
\text { intensifs }\end{array}$ & $\begin{array}{l}\text { Admission du patient à l'unité des soins intensifs au cours de son hospitalisation pour une pneumococcie } \\
\text { invasive, mentionnée dans le dossier médical électronique }\end{array}$ \\
\hline \multicolumn{2}{|l|}{ Résultat } \\
\hline Décès à l'hôpital & $\begin{array}{l}\text { Décès du patient pendant son hospitalisation pour une pneumococcie invasive, mentionné dans son dossier } \\
\text { médical électronique }\end{array}$ \\
\hline \multicolumn{2}{|l|}{ Vaccination } \\
\hline $\begin{array}{l}\text { Vaccination antipneumococcique } \\
\text { antérieure }\end{array}$ & $\begin{array}{l}\text { Vaccination antipneumococcique administrée au cours des cinq dernières années, figurant dans le dossier } \\
\text { médical électronique du patient ou dans le système d'information électronique sur la santé publique }\end{array}$ \\
\hline
\end{tabular}

Abbreviations: $\mathrm{HCV}$, hepatitis $\mathrm{C}$ virus; ICU, intensive care unit; IPD, invasive pneumococcal disease 